Keywords: childhood cancer; bone sarcoma; long-term outcomes; Ewing sarcoma; osteosarcoma; bone cancer; paediatric cancer; late effects

\title{
Long-term adverse outcomes in survivors of childhood bone sarcoma: the British Childhood Cancer Survivor Study
}

M M Fidler ${ }^{1}$, C Frobisher ${ }^{1}$, J Guha ${ }^{1}$, K Wong ${ }^{1}$, J Kelly ${ }^{1}, \mathrm{D}$ L Winter ${ }^{1}$, E Sugden ${ }^{1}, \mathrm{R}$ Duncan ${ }^{2}, \mathrm{~J} \mathrm{Whelan}^{3}$, R C Reulen ${ }^{1}$, M M Hawkins ${ }^{*}, 1$ and on behalf of the British Childhood Cancer Survivor Study (BCCSS) Steering Group

${ }^{1}$ Centre for Childhood Cancer Survivor Studies, School of Health and Population Sciences, Public Health Building, University of Birmingham, Birmingham B15 2TT, UK; ${ }^{2}$ Orthopaedic Department, Royal Hospital for Sick Children, Yorkhill, Glasgow G3 8SJ, UK and ${ }^{3}$ NIHR University College London Hospitals Biomedical Research Centre, London NW1 2PG, UK

Background: With improved survival, more bone sarcoma survivors are approaching middle age making it crucial to investigate the late effects of their cancer and its treatment. We investigated the long-term risks of adverse outcomes among 5 -year bone sarcoma survivors within the British Childhood Cancer Survivor Study.

Methods: Cause-specific mortality and risk of subsequent primary neoplasms (SPNs) were investigated for 664 bone sarcoma survivors. Use of health services, health and marital status, alcohol and smoking habits, and educational qualifications were investigated for survivors who completed a questionnaire.

Results: Survivors were seven times more likely to experience all-cause mortality than expected, and there were substantial differences in risk depending on tumour type. Beyond 25 years follow-up the risk of dying from all-causes was comparable to the general population. This is in contrast to dying before 25 years where the risk was 12.7 -fold that expected. Survivors were also four times more likely to develop a SPN than expected, where the excess was restricted to 5-24 years post diagnosis. Increased healthcare usage and poor health status were also found. Nonetheless, for some psychosocial outcomes survivors were better off than expected.

Conclusions: Up to 25 years after 5-year survival, bone sarcoma survivors are at substantial risk of death and SPNs, but this is greatly reduced thereafter. As $95 \%$ of all excess deaths before 25 years follow-up were due to recurrences and SPNs, increased monitoring of survivors could prevent mortality. Furthermore, bone and breast SPNs should be a particular concern. Since there are variations in the magnitude of excess risk depending on the specific adverse outcome under investigation and whether the survivors were initially diagnosed with osteosarcoma or Ewing sarcoma, risks need to be assessed in relation to these factors. These findings should provide useful evidence for risk stratification and updating clinical follow-up guidelines.

Primary malignant bone sarcomas account for $4.8 \%$ of all childhood cancers in the United Kingdom (Stiller, 2007). Approximately 65 cases occur each year, of which the principal tumour types are osteosarcoma (53\%) and Ewing sarcoma (39\%) (Stiller, 2007). Although the incidence is low, survival after bone sarcoma has increased substantially. Since the 1970s 5-year survival

*Correspondence: Professor MM Hawkins; E-mail: m.m.hawkins@bham.ac.uk

Previously presented at the '13th International Conference on Long-Term Complications of Treatment of Children and Adolescents for Cancer' and 'National Cancer Intelligence Network Cancer Outcomes Conference'.

Received 30 January 2015; revised 9 April 2015; accepted 20 April 2015; published online 19 May 2015

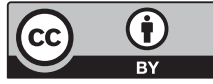


has risen from 23 to $64 \%$ mainly due to the introduction of modern chemotherapy (Stiller, 2007). Consequently, as the number of individuals treated for childhood bone sarcomas increases, it becomes even more important to investigate the risk of the longterm effects of this childhood cancer and its treatment.

This study assessed adverse outcomes among bone sarcoma survivors diagnosed between the ages of 0 and 14 years within the British Childhood Cancer Survivor Study (BCCSS). Key advantages of the BCCSS compared with other studies are that it is a large, population-based cohort with $30.4 \%$ of individuals diagnosed with bone sarcoma surviving to age 45 years at least. Therefore, adverse health and social outcomes beyond 35 years post diagnosis in these childhood cancer survivors can be examined much more satisfactorily than has been possible in previous smaller or nonpopulation-based studies with limited follow-up (Gianinazzi et al, 2013; Jazbec et al, 2004; Cardous-Ubbink et al, 2007; Armstrong et al, 2009; Casagranda et al, 2013). In this study, we investigated the long-term risk of premature mortality, developing a subsequent primary neoplasm, health-care usage, health and marital status, alcohol and smoking habits, and educational attainment among 5-year childhood bone sarcoma survivors.

\section{MATERIALS AND METHODS}

British Childhood Cancer Survivor Study. The BCCSS, which has been described previously in detail (Hawkins et al, 2008), is a population-based cohort comprised of 17980 individuals; it includes 664 bone sarcoma survivors diagnosed with cancer before the age of 15, between 1940 and 1991 in Great Britain, and who have survived at least 5 years. The cohort was ascertained through the National Registry of Childhood Tumours, which has a high estimated level of completeness ( 99\%) (Kroll et al, 2011). Ethical approval for the study was obtained from a Multi-Centre Research Ethics Committee and every Local Research Ethics Committee in Britain.

When treatment exposures within this cohort were investigated across 5-year calendar year bands, we found that before 1976, where our radiotherapy and chemotherapy treatment completeness was $98.4 \%$ and $88.4 \%$, respectively, the majority of bone sarcoma survivors received radiotherapy (76.3\%), with only a small proportion receiving chemotherapy. A distinct change in treatment practice was then observed from 1976 onwards where broadly all survivors received chemotherapy and Ewing sarcoma survivors additionally received radiotherapy. Thus, in order to address the incompleteness of treatment information in more recent diagnosis years, which was due to decreasing availability of recorded radiotherapy and chemotherapy details at the National Registry of Childhood Tumours during this period, our analyses were undertaken for bone sarcoma survivors overall and separately for osteosarcoma and Ewing sarcoma, which serve as proxies for treatment exposures. Therefore, osteosarcoma survivors were likely to have received radiotherapy if diagnosed before 1976 and only chemotherapy if diagnosed from 1976 onwards, whereas all Ewing sarcoma survivors were likely to have received radiotherapy, with only those diagnosed after 1976 additionally receiving chemotherapy. Consequently, those surviving beyond 25 years from 5 -year survival were likely to have only received radiotherapy, whilst those with $<25$ years follow-up were likely to have received only chemotherapy or chemotherapy and radiotherapy depending on tumour type.

Record linkage ascertained outcomes. Deaths and subsequent primary neoplasms (SPNs) were ascertained for the entire BCCSS cohort through record linkage with the National Health Service Information Centre, which includes the population-based national death and cancer registries. This linkage ensures that the BCCSS is notified whenever a survivor has died or developed a SPN. To determine the expected number of deaths or incident cancers, person-years for each sex-specific, age-specific (5-year bands), and calendar year-specific (1-year bands) stratum were multiplied by the corresponding general population rates for specific cause(s) of death and incident cancers occurring throughout England and Wales.

Cause-specific mortality. For our mortality analysis, the death certificate and underlying cause of death, as coded by the Office for National Statistics using the relevant International Classification of Disease, were obtained. The underlying cause of death was then confirmed by a clinician using available medical records. Time at risk started at 5-year survival and continued until individuals exited from risk at the first occurrence of emigration, death, or 31 December 2010 which was the date of the most recent vital status update on the entire cohort from the National Health Service Information Centre. The standardised mortality ratio (SMR) was defined as the ratio of observed to expected number of deaths. The absolute excess risk (AER) was defined as the observed minus the expected number of deaths divided by person-years at risk multiplied by 10000 . Cumulative mortality for a specific cause of death was calculated by treating other causes of death as competing risks.

Subsequent primary neoplasms. Confirmation of all SPNs was undertaken by writing to the relevant clinician(s) to obtain diagnostic reports to confirm site, type and date of diagnosis. Time at risk for a SPN began at 5-year survival and individuals exited from risk at the first occurrence of an SPN, emigration, death, or 31 December 2006 which was the most recent date up to which all potential SPNs had been ascertained and validated. Standardised incidence ratios (SIRs) were calculated as the ratio of observed to expected number of neoplasms. The AERs were calculated as described previously for the mortality analyses. Cumulative incidence for the first occurrence of a SPN was computed treating death as a competing risk.

Questionnaire ascertained outcomes. Health-care usage, health and marital status, alcohol and smoking habits, and educational attainment were obtained via the BCCSS questionnaire. To be eligible to receive the BCCSS questionnaire survivors in the cohort had to be alive and aged at least 16 years at questionnaire send-out (median year 2002). Of the 664 bone sarcoma survivors, 506 survivors met this eligibility criteria and were contacted; amongst survivors who were ineligible, the majority had died before the questionnaire send-out $(n=106)$. Ultimately, $411(81.2 \%)$ returned a completed questionnaire. All comparisons with the general population were adjusted for age and sex. Some outcomes were adjusted further-see tables for details.

Health-care usage. Four types of health-care usage were assessed: talking to a doctor, attending the hospital outpatient department, being hospitalised as a day patient, and being hospitalised as an inpatient. In order to compare health-care use with the general population, the 2002 General Household Survey (GHS) served as the general population sample (Richards et al, 2004). Multivariable generalised estimating equation (GEE) logistic regression modelling was used to calculate odds ratios (ORs) for health-care usage among bone sarcoma survivors compared to that expected from the general population sample (Rebholz et al, 2011).

Psychosocial outcomes. The survivors' education level, smoking history, and alcohol consumption were compared with the general population using the 2002 GHS (Richards et al, 2004) as the reference sample, whereas marital status was compared with the National Marriage Registry (Office for National Statistics, 2002). Multivariable GEE logistic regression was used to compare educational attainment, smoking status, and alcohol use between survivors and the general population sample (Frobisher et al, 2007; 
Frobisher et al, 2008; Frobisher et al, 2010; Lancashire et al, 2010). ORs were calculated using pooled Mantel-Haenszel tests to compare marital status between survivors and the general population sample.

Health status. Version one of the Short Form 36 (SF-36) Health Survey was used to measure self-reported health status by the following eight scales: physical function, role-physical, role-emotional, social functioning, mental health, vitality, pain, and general health perception. External comparisons were conducted using the Oxford Healthy Life Survey (OHLS) as the general population sample. Multivariable linear regression and direct standardisation were used to compare bone sarcoma survivors and the OHLS population.

All analyses were undertaken using Stata 12.1 (StataCorp, College Station, TX, USA). Statistical significance was defined as a two-sided $P$-value $<0.05$.

\section{RESULTS}

Cohort characteristics. Of the 664 bone sarcoma survivors, 309 (46.5\%) were diagnosed with osteosarcoma, 260 (39.2\%) were diagnosed with Ewing sarcoma, 26 (3.9\%) were diagnosed with chondrosarcoma, 48 (7.2\%) were diagnosed with other specified bone sarcomas (e.g., fibromatous neoplasms, giant cell tumours, chordomas, and miscellaneous bone tumours), and 21 (3.2\%) were diagnosed with an unspecified bone sarcoma. The mean age at diagnosis was 10.8 and the average attained age was 39.4 years (Table 1). Osteosarcoma survivors were older at diagnosis and had a higher attained age compared with Ewing sarcoma survivors. Excluding missing information, $60.2 \%$ and $60.0 \%$ of survivors received radiotherapy and chemotherapy, respectively. In general, characteristics of the 411 survivors who returned a questionnaire were similar both overall and by tumour type to the entire BCCSS bone sarcoma cohort, except that only $3.7 \%$ had died by 31 December 2010 subsequent to completing a questionnaire.

\section{Record linkage ascertained data}

Cause-specific mortality. Overall, bone sarcoma survivors experienced seven times (SMR: 7.0, 95\% confidence interval (CI): 5.9-8.3) the number of deaths expected from the general population with 72 (95\% CI: 57.2-85.8) excess deaths per 10000 person-years (Table 2). The largest excess was for neoplastic-related causes in

Table 1. Characteristics of bone sarcoma study population overall and by tumour type

\begin{tabular}{|c|c|c|c|c|c|c|}
\hline \multirow[b]{2}{*}{ Characteristic } & \multicolumn{3}{|c|}{ Available survivors for data linkage $(N=664)$} & \multicolumn{3}{|c|}{ Available questionnaire completed survivors $(N=411)$} \\
\hline & $\begin{array}{c}\text { All } \\
N(\%)\end{array}$ & $\begin{array}{c}\text { Osteosarcoma } \\
n(\%)\end{array}$ & $\begin{array}{c}\text { Ewing sarcoma } \\
n(\%)\end{array}$ & $\begin{array}{c}\text { All } \\
N(\%)\end{array}$ & $\begin{array}{c}\text { Osteosarcoma } \\
n(\%)\end{array}$ & $\begin{array}{c}\text { Ewing sarcoma } \\
n(\%)\end{array}$ \\
\hline \multicolumn{7}{|l|}{ Sex } \\
\hline Male & $345(52.0)$ & $150(48.5)$ & $138(53.1)$ & $200(48.7)$ & $96(47.8)$ & $74(48.1)$ \\
\hline Female & $319(48.0)$ & $159(51.5)$ & $122(46.9)$ & $211(51.3)$ & $105(52.2)$ & $80(52.0)$ \\
\hline \multicolumn{7}{|l|}{ Cancer site } \\
\hline Upper limbs & $92(13.9)$ & $34(11.0)$ & $48(18.5)$ & $56(13.7)$ & $21(10.5)$ & $32(20.8)$ \\
\hline Lower limbs & $436(65.7)$ & $262(84.8)$ & $132(50.8)$ & $273(66.5)$ & $170(84.6)$ & $75(48.7)$ \\
\hline Bones of skull and face & $42(6.4)$ & $5(1.6)$ & $10(3.9)$ & $26(6.3)$ & $4(2.0)$ & $6(3.9)$ \\
\hline Vertebral column & $24(3.6)$ & $3(1.0)$ & $15(5.8)$ & $13(3.2)$ & $3(1.5)$ & $7(4.6)$ \\
\hline Rib, sternum, clavicle & $28(4.2)$ & $1(0.3)$ & $25(9.6)$ & $20(4.9)$ & $1(0.5)$ & $17(11.0)$ \\
\hline Pelvic, sacrum, coccyx & $33(5.0)$ & $2(0.7)$ & $27(10.4)$ & $19(4.6)$ & $2(1.0)$ & $15(9.7)$ \\
\hline Other & $9(1.4)$ & $2(0.7)$ & $3(1.2)$ & $4(1.0)$ & $0(0)$ & $2(1.3)$ \\
\hline \multicolumn{7}{|l|}{ Age at diagnosis } \\
\hline Mean (range) & $10.8(0.1-15.0)$ & $11.5(2.3-15.0)$ & $10.2(1.5-15.0)$ & $10.8(1.3-15.0)$ & $11.6(3.2-15.0)$ & $10.0(2.0-15.0)$ \\
\hline $0-4$ years & $40(6.0)$ & $8(2.6)$ & $22(8.5)$ & $22(5.4)$ & $4(2.0)$ & $13(8.4)$ \\
\hline $5-9$ years & $185(27.9)$ & $74(24.0)$ & $84(32.3)$ & $122(29.7)$ & $46(22.9)$ & $58(37.7)$ \\
\hline $10-14$ years & $439(66.1)$ & $227(73.5)$ & $154(59.2)$ & $267(65.0)$ & $151(75.1)$ & $83(53.9)$ \\
\hline \multicolumn{7}{|l|}{ Attained age at exit } \\
\hline Mean (range) & $39.4(7.5-76.8)^{a}$ & $40.9(10.0-71.9)^{\mathrm{a}}$ & $35.7(7.5-65.2)^{\mathrm{a}}$ & $43.3(22.4-76.8)^{b}$ & $44.6(22.9-71.9)^{b}$ & $39.4(22.4-65.2)^{b}$ \\
\hline $16-24$ years & $89(13.4)$ & $31(10.0)$ & $47(18.1)$ & $71(17.3)$ & $23(11.4)$ & $43(27.9)$ \\
\hline $25-34$ years & $155(23.3)$ & $62(20.1)$ & $78(30.0)$ & 169 (41.1) & $83(41.3)$ & $72(46.8)$ \\
\hline $35-44$ years & $218(32.8)$ & $108(35.0)$ & $90(34.6)$ & $92(22.4)$ & $53(26.4)$ & 25 (16.2) \\
\hline $45+$ years & $202(30.4)$ & $108(35.0)$ & 45 (17.3) & 79 (19.2) & $42(20.9)$ & $14(9.1)$ \\
\hline \multicolumn{7}{|l|}{ Radiotherapy ${ }^{c}$} \\
\hline No & 201 (39.8) & $150(59.3)$ & $17(9.9)$ & $125(40.3)$ & $98(58.3)$ & $10(10.5)$ \\
\hline Yes & $304(60.2)$ & $103(40.7)$ & $154(90.1)$ & $185(59.7)$ & $70(41.7)$ & $85(89.5)$ \\
\hline \multicolumn{7}{|l|}{ Chemotherapyc } \\
\hline No & $195(40.0)$ & 93 (37.5) & $40(23.8)$ & $109(36.7)$ & $58(35.6)$ & $16(17.2)$ \\
\hline Yes & $292(60.0)$ & $155(62.5)$ & $128(76.2)$ & $188(63.3)$ & $105(64.4)$ & 77 (82.8) \\
\hline \multicolumn{7}{|l|}{ Surgeryc } \\
\hline No & $160(31.3)$ & $34(13.2)$ & $109(63.4)$ & $93(29.4)$ & $19(11.1)$ & $62(64.6)$ \\
\hline Yes & $352(68.8)$ & $223(86.8)$ & 63 (36.6) & $223(70.6)$ & $152(88.9)$ & $34(35.4)$ \\
\hline \multicolumn{7}{|l|}{ Vital status $^{a}$} \\
\hline Alive & $533(80.3)$ & $256(82.9)$ & $203(78.1)$ & $396(96.4)$ & $193(96.0)$ & $150(97.4)$ \\
\hline Dead & $131(19.7)$ & 53 (17.2) & 57 (21.9) & $15(3.7)$ & $8(4.0)$ & $4(2.6)$ \\
\hline \multicolumn{7}{|c|}{$\begin{array}{l}\text { a Age at } 31 \text { December } 2010 \text { or death/embarkation (if before } 31 \text { December 2010)—relevant to the mortality analyses. } \\
\text { bAge at questionnaire completion-relevant to outcomes measured by the questionnaire. } \\
\text { c Missing data: radiotherapy (all data linkage) }=139 \text {, radiotherapy (all questionnaire) }=101 \text {; chemotherapy (all data linkage) =157, chemotherapy (all questionnaire)=114; surgery (all dat } \\
\text { linkage) }=152 \text {, Surgery (all questionnaire) }=95 \text {. }\end{array}$} \\
\hline
\end{tabular}



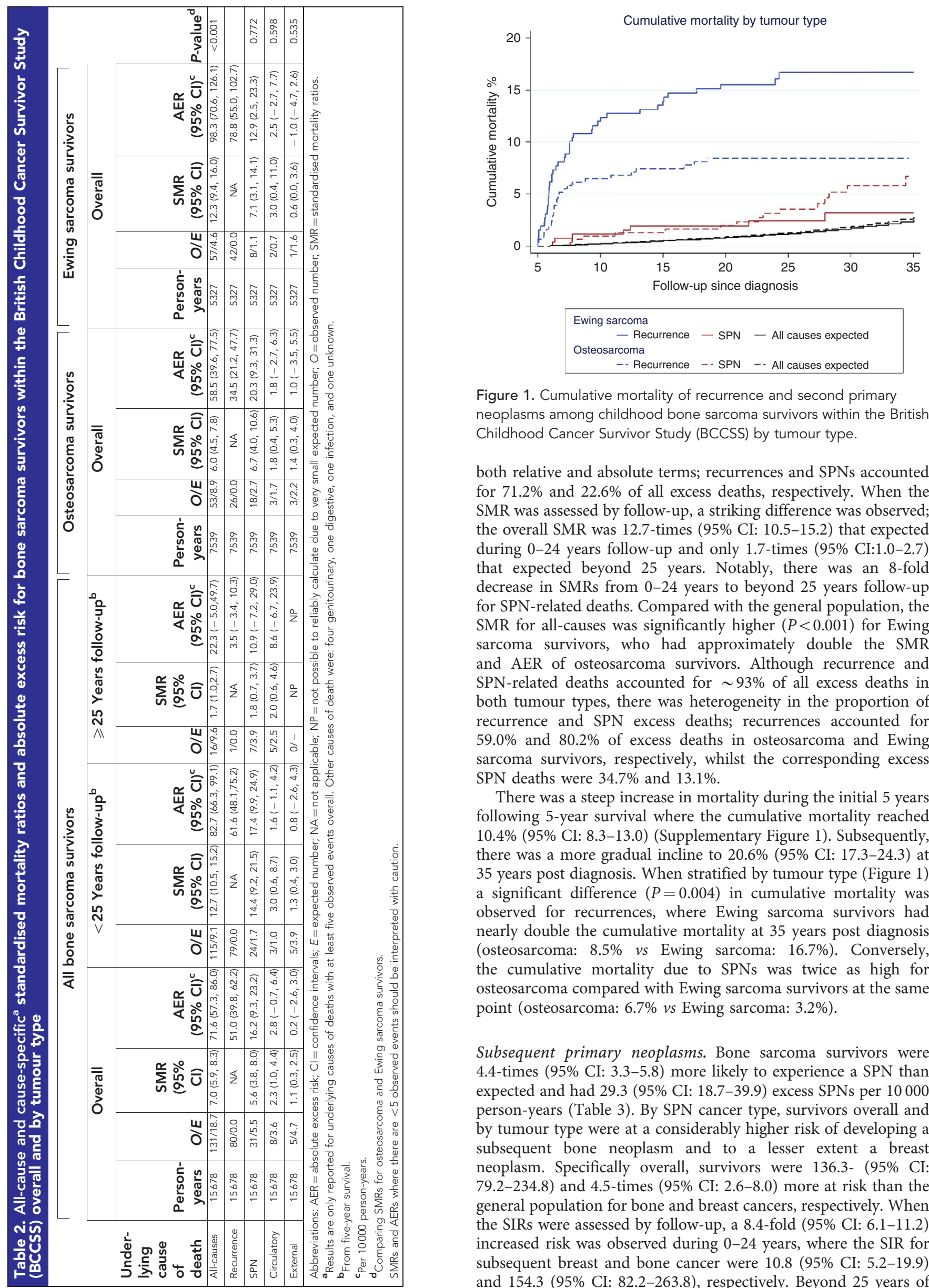

$$
\begin{aligned}
& \text { Ewing sarcoma } \\
& \text { - Recurrence - SPN - All causes expected } \\
& \text { Osteosarcoma } \\
& \text {-- Recurrence --SPN -- All causes expected }
\end{aligned}
$$

Figure 1. Cumulative mortality of recurrence and second primary neoplasms among childhood bone sarcoma survivors within the British Childhood Cancer Survivor Study (BCCSS) by tumour type.

both relative and absolute terms; recurrences and SPNs accounted for $71.2 \%$ and $22.6 \%$ of all excess deaths, respectively. When the SMR was assessed by follow-up, a striking difference was observed; the overall SMR was 12.7-times (95\% CI: 10.5-15.2) that expected during 0-24 years follow-up and only 1.7-times (95\% CI:1.0-2.7) that expected beyond 25 years. Notably, there was an 8-fold decrease in SMRs from 0-24 years to beyond 25 years follow-up for SPN-related deaths. Compared with the general population, the SMR for all-causes was significantly higher $(P<0.001)$ for Ewing sarcoma survivors, who had approximately double the SMR and AER of osteosarcoma survivors. Although recurrence and SPN-related deaths accounted for $\sim 93 \%$ of all excess deaths in both tumour types, there was heterogeneity in the proportion of recurrence and SPN excess deaths; recurrences accounted for $59.0 \%$ and $80.2 \%$ of excess deaths in osteosarcoma and Ewing sarcoma survivors, respectively, whilst the corresponding excess SPN deaths were $34.7 \%$ and $13.1 \%$.

There was a steep increase in mortality during the initial 5 years following 5-year survival where the cumulative mortality reached 10.4\% (95\% CI: 8.3-13.0) (Supplementary Figure 1). Subsequently, there was a more gradual incline to $20.6 \%$ (95\% CI: $17.3-24.3)$ at 35 years post diagnosis. When stratified by tumour type (Figure 1) a significant difference $(P=0.004)$ in cumulative mortality was observed for recurrences, where Ewing sarcoma survivors had nearly double the cumulative mortality at 35 years post diagnosis (osteosarcoma: $8.5 \%$ vs Ewing sarcoma: 16.7\%). Conversely, the cumulative mortality due to SPNs was twice as high for osteosarcoma compared with Ewing sarcoma survivors at the same point (osteosarcoma: 6.7\% vs Ewing sarcoma: 3.2\%).

Subsequent primary neoplasms. Bone sarcoma survivors were 4.4-times (95\% CI: 3.3-5.8) more likely to experience a SPN than expected and had 29.3 (95\% CI: 18.7-39.9) excess SPNs per 10000 person-years (Table 3). By SPN cancer type, survivors overall and by tumour type were at a considerably higher risk of developing a subsequent bone neoplasm and to a lesser extent a breast neoplasm. Specifically overall, survivors were 136.3- (95\% CI: 79.2-234.8) and 4.5-times (95\% CI: 2.6-8.0) more at risk than the general population for bone and breast cancers, respectively. When the SIRs were assessed by follow-up, a 8.4 -fold (95\% CI: 6.1-11.2) increased risk was observed during 0-24 years, where the SIR for subsequent breast and bone cancer were 10.8 (95\% CI: 5.2-19.9) and 154.3 (95\% CI: 82.2-263.8), respectively. Beyond 25 years of 


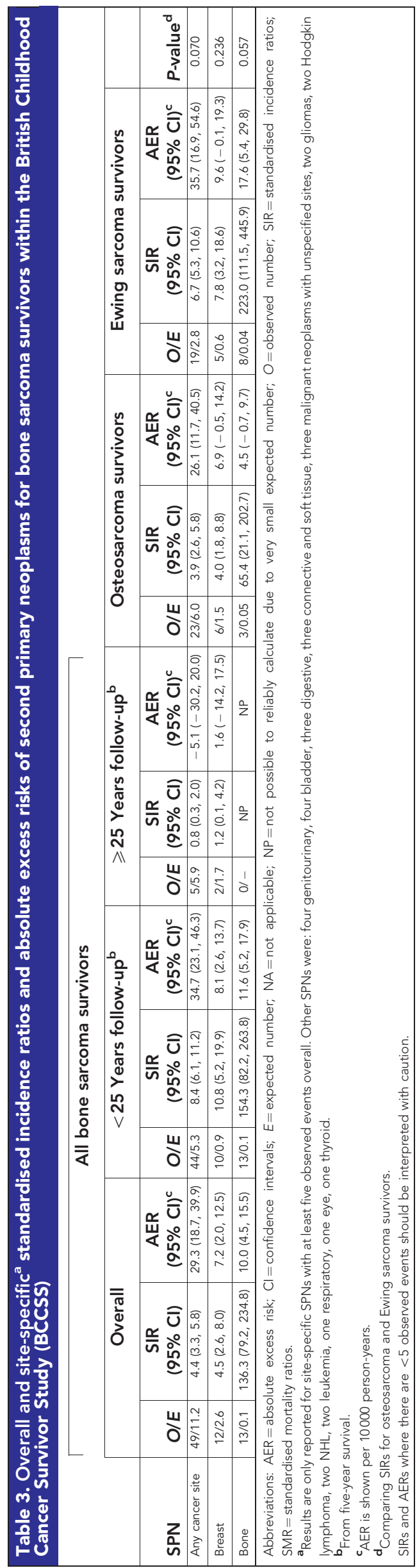

follow-up, the SIR for any SPN was not significantly higher in survivors than expected from the general population.

There was a continuous and steady increase in cumulative incidence for SPNs over follow-up, ultimately reaching 8.3\% (95\% CI: 5.9-11.2) at 30 years post diagnosis (Supplementary Figure 2). When stratified by tumour type, the cumulative incidence curves were nearly identical to each other and to bone sarcoma survivors overall $(P>0.05)$.

\section{Questionnaire ascertained data}

Health-care usage. Compared with the general population sample, bone sarcoma survivors were almost three times (OR: 2.9, 95\% CI: 2.3-3.7) more likely to have visited an outpatient hospital department in the previous three months (Table 4). Survivors were also over twice (OR: 2.4, 95\% CI: 1.7-3.4) more likely to be hospitalised as an inpatient during the previous year than the general population sample. When analysed by tumour type, both osteosarcoma and Ewing sarcoma survivors had significantly higher odds of attending the hospital as an outpatient or inpatient than expected.

Psychosocial outcomes. Bone sarcoma survivors overall were comparable to the general population sample for being evermarried, a current drinker, or consuming harmful amounts of alcohol (Table 4). Survivors were, however, significantly less likely to be a current smoker (OR: 0.6, 95\% CI: 0.5-0.8) and consume alcohol over recommendations (OR: 0.7, 95\% CI: 0.5-0.9) than the general population sample. Compared to that expected, survivors performed well in obtaining educational qualifications and were $70 \%$ more likely to have obtained at least O-levels (OR: 1.7, 95\% CI: 1.3-2.1). When analysed by tumour type, the odds for each psychosocial outcome were comparable to the overall finding.

Health status. Compared with the general population sample, bone sarcoma survivors overall were significantly more limited in all SF-36 scales with the exception of role-emotional (Figure 2). The most notable differences occurred in physical function, rolephysical, and pain. For the individual components of the physical function scale (Supplementary Figure 3), 54\% and 61\% of survivors were limited in 'moderate activities' and 'walking more than one mile' compared with the $8 \%$ and $11 \%$ expected from the general population sample, respectively. In the role-physical scale (Supplementary Figure 4), the largest difference between the survivors and general population sample was in 'being limited in the kind of work and activities', although all component questions reported at least a $10 \%$ deficit. Finally, for the pain scale (Supplementary Figure 5), survivors reported more bodily pain $(12 \%$ vs $5 \%)$ and more pain interference ( $16 \%$ vs $5 \%$ ) during the past 4 weeks compared with the general population sample.

When stratified by tumour type, osteosarcoma survivors were assessed additionally by amputation status, where only arm or leg amputations as a form of initial treatment for the first primary tumour were included. Compared with the general population sample, osteosarcoma amputee survivors reported being the most limited in all scales relative to osteosarcoma non-amputees and Ewing sarcoma survivors, with a significantly $(P<0.001)$ higher disadvantage in physical function (Figure 2).

\section{DISCUSSION}

This is the first large-scale population-based study to provide a comprehensive description of long-term health and social outcomes among a large cohort of 5-year bone sarcoma survivors, both overall and by tumour type, beyond 35 years post diagnosis. Mortality estimates in this cohort were elevated seven times that expected and 
Table 4. Percentages and odds ratios (with corresponding $95 \% \mathrm{Cls}$ ) for the likelihood of use of health services and psychosocial outcomes in bone sarcoma survivors within the British Childhood Cancer Survivor Study (BCCSS) compared with the general population of Britain

\begin{tabular}{|c|c|c|c|c|}
\hline & UK norms (ref) & $\begin{array}{c}\text { Bone sarcoma overall } \\
\text { OR }(95 \% \mathrm{Cl})\end{array}$ & $\begin{array}{l}\text { Osteosarcoma } \\
\text { OR (95\% Cl) }\end{array}$ & $\begin{array}{l}\text { Ewing sarcoma } \\
\text { OR }(95 \% \mathrm{Cl})\end{array}$ \\
\hline \multicolumn{5}{|l|}{ Marital Status ${ }^{a}$} \\
\hline Males ever-married & 1.0 & $0.7(0.5,1.0)$ & $1.0(0.6,1.6)$ & $0.7(0.4,1.3)$ \\
\hline Females ever-married & 1.0 & $0.8(0.6,1.1)$ & $0.8(0.5,1.2)$ & $0.9(0.6,1.4)$ \\
\hline \multicolumn{5}{|l|}{ Education $^{\text {b }}$} \\
\hline University degree or higher & 1.0 & $1.2(1.0,1.6)$ & $1.5(1.1,2.1)$ & $1.0(0.8,1.5)$ \\
\hline Teaching qualification or higher & 1.0 & $1.1(0.9,1.4)$ & $1.3(1.0,1.7)$ & $1.0(0.7,1.4)$ \\
\hline A-levels or higher ${ }^{c}$ & 1.0 & $1.2(1.0,1.5)$ & $1.2(0.9,1.6)$ & $1.1(0.8,1.5)$ \\
\hline O-levels or higher ${ }^{d}$ & 1.0 & $1.7(1.3,2.1)$ & $1.8(1.2,2.6)$ & $2.0(1.2,3.1)$ \\
\hline \multicolumn{5}{|l|}{ Alcohol $^{e}$} \\
\hline Current drinker & 1.0 & $0.8(0.6,1.1)$ & $1.0(0.6,1.6)$ & $0.8(0.5,1.3)$ \\
\hline Consuming over recommendations & 1.0 & $0.7(0.5,0.9)$ & $0.7(0.5,1.1)$ & $0.5(0.3,0.9)$ \\
\hline Consuming harmful amounts & 1.0 & $0.7(0.4,1.1)$ & $0.6(0.3,1.4)$ & $0.7(0.3,1.5)$ \\
\hline \multicolumn{5}{|l|}{ Smoking ${ }^{\mathrm{e}}$} \\
\hline Current smoker & 1.0 & $0.6(0.5,0.8)$ & $0.7(0.5,1.0)$ & $0.6(0.4,0.9)$ \\
\hline \multicolumn{5}{|l|}{ Use of health services ${ }^{f}$} \\
\hline Talked to a doctor ${ }^{9}$ & 1.0 & $1.2(0.9,1.6)$ & $1.3(0.9,1.8)$ & $1.2(0.8,1.8)$ \\
\hline Attended as outpatient ${ }^{\mathbf{9}}$ & 1.0 & $2.9(2.3,3.7)$ & $2.9(2.1,4.0)$ & $3.2(2.2,4.7)$ \\
\hline Attended as day patient ${ }^{\mathrm{h}}$ & 1.0 & $1.1(0.7,1.5)$ & $1.2(0.7,1.9)$ & $1.0(0.6,1.8)$ \\
\hline Attended as inpatient ${ }^{\text {h }}$ & 1.0 & $2.4(1.7,3.4)$ & $2.5(1.6,3.9)$ & $2.8(1.7,4.7)$ \\
\hline \multicolumn{5}{|c|}{ 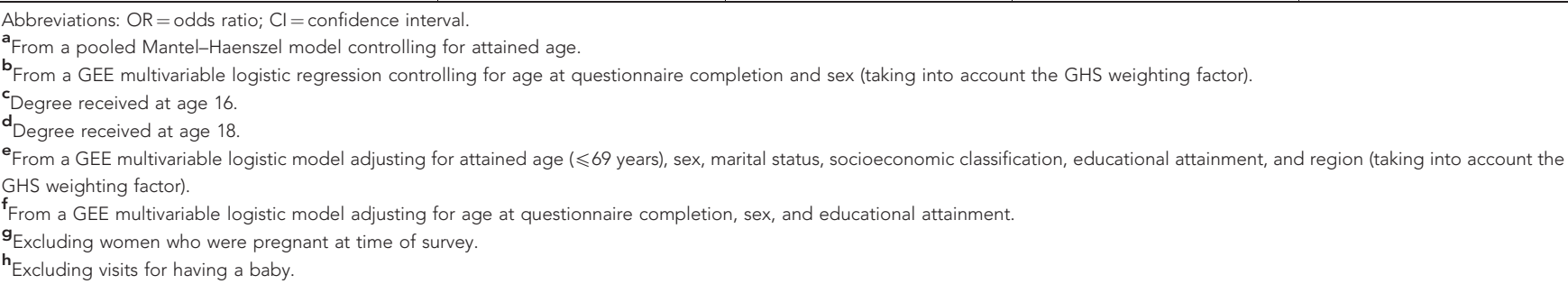 } \\
\hline
\end{tabular}

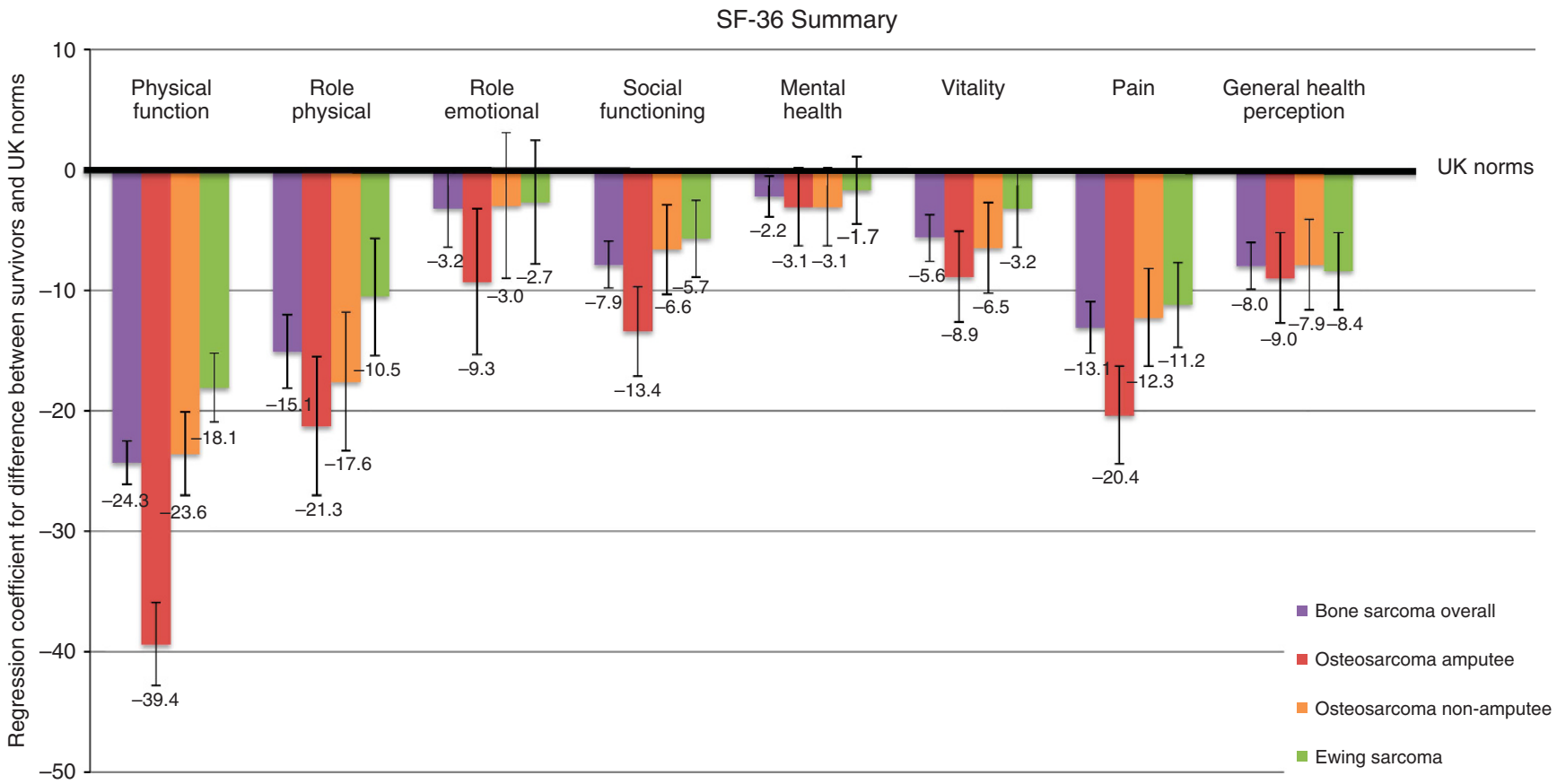

Figure 2. Sex and age adjusted regression coefficients and corresponding 95\% confidence intervals for differences in SF-36 health status scales between bone sarcoma, osteosarcoma amputees, osteosarcoma non-amputees, and Ewing sarcoma survivors vs UK norms.

varied significantly between tumour types, which were consistent with previous findings of a large-scale US study (Armstrong et al, 2009). Past studies have also shown the principal cause of death was neoplastic-related (MacArthur et al, 2007; Ginsberg et al, 2010; Nagarajan et al, 2011). However, to our knowledge, this is the first study that has shown substantial differences when comparing excess 
and cumulative mortality between tumour types; osteosarcoma survivors had double the cumulative mortality for SPNs compared with Ewing sarcoma and Ewing sarcoma survivors had double the cumulative mortality for recurrences compared with osteosarcoma at 35 years post diagnosis. The osteosarcoma survivors in this study were much more likely to have an amputation than Ewing sarcoma, which may partially explain why osteosarcoma survivors were less likely to have recurred (Grimer et al, 2002). Additionally, due to the extended follow-up available, this is the first study to show that beyond 25 years follow-up the risk of dying from all-causes is comparable to the general population and unlikely to exceed 2.7-fold that expected. This is in contrast to dying before 25 years of follow-up, where the risk is 12.7 -fold that expected. This provides important evidence for clinicians who monitor survivors treated in similar decades to those included in the BCCSS. A possible explanation for this striking absence or low risk of excess mortality with extended follow-up may relate to our previous observation that, as the overall cohort of childhood cancer survivors ages, a large proportion of excess deaths are attributed to SPNs (Reulen et al, 2010), particularly breast, digestive, genitourinary, and lung carcinomas. Although carcinomas of these sites are common cancers of adulthood in the general population, in childhood cancer survivors they are principally caused by direct radiotherapy exposure (Reulen et al, 2011). As $80 \%$ of the bone sarcomas included here were diagnosed in the limb, there is unlikely to have been much direct exposure from radiotherapy to tissues of these sites due to the lack of proximity of the radiotherapy fields.

The overall and tumour type-specific SIRs for SPNs were consistent with previous studies (Magnani et al, 1996; CardousUbbink et al, 2007; Inskip and Curtis, 2007; Friedman et al, 2010). Additionally, our findings are consistent with previous literature in that the most common SPN was breast cancer for osteosarcoma survivors and bone cancer for Ewing sarcoma survivors (Inskip and Curtis, 2007). Due to the extended follow-up available, this is the first study to show that the risk of developing SPNs was 9.9-fold the expected during 0-24 years follow-up and comparable to the general population beyond 25 years follow-up, where it was unlikely to exceed 2.0-fold that expected. Notably, all thirteen bone cancers occurred before 25 years follow-up, nine of which developed inside or on the edge of tissue directly irradiated to treat the original bone sarcoma and one in a survivor diagnosed with a p53 mutation. This corresponds with our previous work that found that bone cancer is the most common SPN after a first primary bone sarcoma (Reulen et al, 2011), which is principally attributable to exposure of the SPN site to radiation during treatment for the first cancer (Tucker et al, 1987; Hawkins et al, 1996; Schwartz et al, 2014). Of the two breast cancers observed subsequent to 25 years follow-up, both developed in survivors previously treated for a bone sarcoma of a lower limb with unknown p53 status.

Consistent with other studies (Eiser et al, 2001; Hudson et al, 2003; Zeltzer et al, 2008), we reported that survivors were severely limited in health status, in particular physical function and pain. Although previous studies have suggested that health status among amputees is generally similar to non-amputees (Eiser and Grimer, 1999; Nagarajan et al, 2004; Paul, 2008; Eiser, 2009; Nagarajan et al, 2009; Barrera et al, 2012), we found that osteosarcoma amputees reported the worst health status for all scales, with significantly higher limitations in physical function relative to osteosarcoma non-amputees and Ewing sarcoma (95\% of which were non-amputees) survivors.

Although we report here on appreciable proportions of the bone sarcoma survivors experiencing detrimental effects to their health, many of their social outcomes were favourable. In fact, our findings suggest that survivors were more likely than expected to obtain some types of educational qualification and less likely to be a current smoker than expected from the general population.
Current guidelines and recommendations. The Bone Cancer Research Trust currently recommends yearly follow-up after 5-year survival (Newby and Unsworth, 2013a, 2013b). From the evidence presented here, $74 \%$ and $21 \%$ of all excess deaths before 25 years of follow-up were due to recurrence and SPN, respectively, and therefore monitoring of survivors for recurrences and SPNs, particularly during the period $5-10$ years post diagnosis where risk of recurrence is highest, could help prevent premature mortality. Bone and breast SPNs should also be a particular concern and regular follow-up should be provided, particularly in the period 0-24 years following 5-year survival for bone SPNs. Factsheets given to childhood cancer survivors could further expand upon the risk of recurrence and SPNs and the potential for early diagnosis by detailing more precisely signs and symptoms relating to bone and breast neoplasms. Furthermore, the substantial excess risks of specific physical limitations and pain are likely to be useful for risk stratification and possible interventions that seek to reduce morbidity and the practical difficulties that survivors may face.

Limitations. Although the findings in this paper may not be generalisable for children diagnosed after 1991, the purpose of this study was to address the long-term, beyond 35 years post diagnosis, outcomes that childhood bone sarcoma survivors are currently facing. We acknowledge reassessment is necessary and recommend further analyses to be conducted on the recently extended BCCSS cohort, which includes 5-year survivors diagnosed from 1992 to 2006. Furthermore, as a large proportion of bone sarcoma diagnoses occur in individuals aged over 14 years, we recommend further analyses on adverse outcomes to be assessed using the Teenage and Young Adult Cancer Survivor Study (TYACSS), which we have established recently and includes all 5-year survivors diagnosed from age 15-39 in England and Wales between 1970 and 2006. Finally, a potential limitation of our study is the lack of detailed treatment information. Although we report a large reduction in excess mortality and SPNs beyond 25 years follow-up, those followed-up for $<25$ years are more likely to be treated differently due to the introduction of chemotherapy. Thus, reassessment of these more recently diagnosed individuals is essential in order to determine whether the decreases in risk reported in this study remain with newer treatment practices. Nevertheless, due to our population-based design, the evidence presented here provides a reliable and unbiased basis to update clinical follow-up guidelines in relation to bone sarcoma survivors diagnosed before age 15 and treated before 1992 in Great Britain by using cancer diagnosis as a proxy.

\section{CONCLUSIONS}

In conclusion, childhood bone sarcoma survivors diagnosed between 1940 and 1991 in this cohort are at substantial risk of death and SPNs up to 25 years after 5-year survival, but the risk is greatly reduced thereafter. Survivors additionally face difficulties in daily life due to their excess prevalence of poor physical health status. As there are variations in the degree of excess depending on the specific outcome and whether they survived osteosarcoma or Ewing sarcoma, risk needs to be assessed in a stratified way. These findings should provide useful evidence for risk stratification, updating clinical follow-up guidelines, and possible intervention studies.

\section{ACKNOWLEDGEMENTS}

The British Childhood Cancer Survivor Study (BCCSS) is a national collaborative undertaking guided by a Steering Group that 
comprises Professor Douglas Easton (chair), Professor Michael Hawkins, Dr Helen Jenkinson, Dr Meriel Jenney, Dr Raoul Reulen, Professor Kathryn Pritchard-Jones, Professor Michael Stevens, Dr Elaine Sugden, Dr Andrew Toogood, and Dr Hamish Wallace. The BCCSS benefits from the contributions of the Officers, Centers, and individual members of the Children's Cancer and Leukemia Group and the Regional Pediatric Cancer Registries. The BCCSS acknowledges the collaboration of the Office for National Statistics, the General Register Office for Scotland, the Welsh Cancer Intelligence and Surveillance Unit, the National Health Service Information Centre, the regional cancer registries, health authorities, and area health boards for providing general practitioner names and addresses and the general practitioners nationwide who facilitated direct contact with survivors. We are particularly thankful to all survivors who completed a 40-page questionnaire and all General Practitioners who returned consent forms. The BCCSS would not have been possible without the support of our funders: University of Birmingham, Cancer Research UK, the Kay Kendall Leukaemia Fund, and the European Commission to whom we offer our profound thanks. Finally thanks to all BCCSS staff who have given many years of dedicated work to bring the BCCSS to fruition. This work was supported by grant number C386/A10422 from Cancer Research UK; the Kay Kendall Leukaemia Fund; PanCareSurFup, European 7th Framework Programme. Raoul C. Reulen is funded by the National Institute for Health Research.

\section{CONFLICT OF INTEREST}

The authors declare no conflict of interest.

\section{DISCLAIMER}

The views expressed in this publication are those of the author(s) and not necessarily those of the NHS, the National Institute for Health Research or the Department of Health.

\section{REFERENCES}

Armstrong GT, Liu Q, Yasui Y, Neglia JP, Leisenring W, Robison LL, Mertens AC (2009) Late mortality among 5-year survivors of childhood cancer: a summary from the Childhood Cancer Survivor Study. J Clin Oncol 27(14): $2328-2338$.

Barrera M, Teall T, Barr R, Silva M, Greenberg M (2012) Health related quality of life in adolescent and young adult survivors of lower extremity bone tumors. Pediatr Blood Cancer 58(2): 265-273.

Cardous-Ubbink MC, Heinen RC, Bakker PJM, van den Berg H, Oldenburger F, Caron HN, Voûte PA, van Leeuwen FE (2007) Risk of second malignancies in long-term survivors of childhood cancer. Eur J Cancer 43(2): 351-362.

Casagranda L, Trombert-Paviot B, Faure-Conter C, Bertrand Y, Plantaz D, Berger C (2013) Self-reported and record-collected late effects in long-term survivors of childhood cancer: a population-based cohort study of the childhood cancer registry of the Rhone-Alpes region (ARCERRA). Pediatr Hematol Oncol 30(3): 195-207.

Eiser C (2009) Assessment of health-related quality of life after bone cancer in young people: easier said than done. Eur J Cancer 45(10): 1744-1747.

Eiser C, Darlington A-SE, Stride CB, Grimer R (2001) Quality of life implications as a consequence of surgery: limb salvage, primary and secondary amputation. Sarcoma 5(4).

Eiser C, Grimer RJ (1999) Quality of life in survivors of a primary bone tumour: a systematic review. Sarcoma 3(3-4): 183-190.

Friedman DL, Whitton J, Leisenring W, Mertens AC, Hammond S, Stovall M, Donaldson SS, Meadows AT, Robison LL, Neglia JP (2010) Subsequent neoplasms in 5-year survivors of childhood cancer: the Childhood Cancer Survivor Study. J Natl Cancer Inst 102(14): 1083-1095.
Frobisher C, Lancashire ER, Reulen RC, Winter DL, Stevens MC, Hawkins MM (2010) Extent of alcohol consumption among adult survivors of childhood cancer: the British Childhood Cancer Survivor Study. Cancer Epidemiol Biomarkers Prev 19(5): 1174-1184.

Frobisher C, Lancashire ER, Winter DL, Jenkinson HC, Hawkins MM (2007) Long-term population-based marriage rates among adult survivors of childhood cancer in Britain. Int J Cancer 121(4): 846-855.

Frobisher C, Winter DL, Lancashire ER, Reulen RC, Taylor AJ, Eiser C, Stevens MC, Hawkins MM (2008) Extent of smoking and age at initiation of smoking among adult survivors of childhood cancer in Britain. J Natl Cancer Inst 100(15): 1068-1081.

Gianinazzi ME, Rueegg CS, Wengenroth L, Bergstraesser E, Rischewski J, Ammann RA, Kuehni CE, Michel G. for Swiss Pediatric Oncology Group (2013) Adolescent survivors of childhood cancer: are they vulnerable for psychological distress? Psychooncology 22(9): 2051-2058.

Ginsberg JP, Goodman P, Leisenring W, Ness KK, Meyers PA, Wolden SL, Smith SM, Stovall M, Hammond S, Robison LL, Oeffinger KC (2010) Long-term survivors of childhood Ewing sarcoma: report from the childhood cancer survivor study. J Natl Cancer Inst 102(16): 1272-1283.

Grimer RJ, Taminiau AM, Cannon SR (2002) Surgical outcomes in osteosarcoma. J Bone Joint Surg 84-B(3): 395-400.

Hawkins MM, Lancashire ER, Winter DL, Frobisher C, Reulen RC, Taylor AJ, Stevens MC, Jenney M (2008) The British Childhood Cancer Survivor Study: objectives, methods, population structure, response rates and initial descriptive information. Pediatr Blood Cancer 50(5): 1018-1025.

Hawkins MM, Wilson LM, Burton HS, Potok MH, Winter DL, Marsden HB, Stovall MA (1996) Radiotherapy, alkylating agents, and risk of bone cancer after childhood cancer. J Natl Cancer Inst 88(5): 270-278.

Hudson MM, Mertens AC, Yasui Y, Hobbie W, Chen H, Gurney JG, Yeazel M, Recklitis CJ, Marina N, Robison LR, Oeffinger KC. Childhood Cancer Survivor Study Investigators (2003) Health status of adult long-term survivors of childhood cancer: a report from the childhood cancer survivor study. JAMA 290(12): 1583-1592.

Inskip PD, Curtis RE (2007) New malignancies following childhood cancer in the United States, 1973-2002. Int J Cancer 121(10): 2233-2240.

Jazbec J, Ećimović P, Jereb B (2004) Second neoplasms after treatment of childhood cancer in Slovenia. Pediatr Blood Cancer 42(7): 574-581.

Kroll ME, Murphy MF, Carpenter LM, Stiller CA (2011) Childhood cancer registration in Britain: capture-recapture estimates of completeness of ascertainment. Br J Cancer 104(7): 1227-1233.

Lancashire ER, Frobisher C, Reulen RC, Winter DL, Glaser A, Hawkins MM (2010) Educational attainment among adult survivors of childhood cancer in Great Britain: a population-based cohort study. J Natl Cancer Inst 102(4): 254-270.

MacArthur AC, Spinelli JJ, Rogers PC, Goddard KJ, Abanto ZU, McBride ML (2007) Mortality among 5-year survivors of cancer diagnosed during childhood or adolescence in British Columbia, Canada. Pediatr Blood Cancer 48(4): 460-467.

Magnani C, Terracini B, Cordero Di Montezemolo L, Gallone G, Luzzatto L, Mosso ML, Pastore G, Rosso P (1996) Incidence of second primary malignancies after a malignant tumor in childhood: a population-based survey in Piedmont (Italy). Int J Cancer 67(1): 6-10.

Nagarajan R, Clohisy DR, Neglia JP, Yasui Y, Mitby PA, Sklar C, Finklestein JZ, Greenberg M, Reaman GH, Zeltzer L, Robison LL (2004) Function and quality-of-life of survivors of pelvic and lower extremity osteosarcoma and Ewing's sarcoma: the Childhood Cancer Survivor Study. Br J Cancer 91(11): 1858-1865.

Nagarajan R, Kamruzzaman A, Ness KK, Marchese VG, Sklar C, Mertens A, Yasui Y, Robison LL, Marina N (2011) Twenty years of follow-up of survivors of childhood osteosarcoma: a report from the Childhood Cancer Survivor Study. Cancer 117(3): 625-634.

Nagarajan R, Mogil R, Neglia JP, Robison LL, Ness KK (2009) Self-reported global function among adult survivors of childhood lower-extremity bone tumors: a report from the Childhood Cancer Survivor Study (CCSS). J Cancer Surviv 3(1): 59-65.

Newby J, Unsworth H (2013a) What you need to know about primary bone cancer: Ewing's sarcoma. Edition II.: Bone Cancer Research Trust.

Newby J, Unsworth H (2013b) What you need to know about primary bone cancer: Osteosarcoma. Edition II.: Bone Cancer Research Trust.

Office for National Statistics (2002) Marriage, Divorce and Adoption Statistics, England and Wales (Series FM2).

Paul SJ (2008) Long term quality-of-life outcomes in pediatric bone cancer: a systematic review. J Nursing Student Res 1(2). 
Rebholz CE, Reulen RC, Toogood AA, Frobisher C, Lancashire ER, Winter DL, Kuehni CE, Hawkins MM (2011) Health care use of long-term survivors of childhood cancer: the British Childhood Cancer Survivor Study. J Clin Oncol 29(31): 4181-4188.

Reulen R, Frobisher C, Winter DL, Kelly J, Lancashire ER, Stiller CA, Pritchard-Jones K, Jenkinson HC, Hawkins MM. British Childhood Cancer Survivor Study Steering Group (2011) Long-term risks of subsequent primary neoplasms among survivors of childhood cancer. JAMA 305(22): 2311-2319.

Reulen RC, Winter DL, Frobisher C, Lancashire ER, Stiller CA, Jenney ME, Skinner R, Stevens MC, Hawkins MM (2010) Long-term causespecific mortality among survivors of childhood cancer. JAMA 304(2): 172-179.

Richards LFK, Roberts C, Fletcher L, Goddard E (2004) Living in Britain. No 31. Results from the 2002 General Household Survey. The Stationery Office: London, UK.

Schwartz B, Benadjaoud M, Cléro E, Haddy N, El-Fayech C, Guibout C, Teinturier C, Oberlin O, Veres C, Pacquement H, Munzer M, N'Guyen T, Bondiau P-Y, Berchery D, Laprie A, Hawkins M, Winter D, Lefkopoulos
D, Chavaudra J, Rubino C, Diallo I, Bénichou J, de Vathaire F (2014) Risk of second bone sarcoma following childhood cancer: role of radiation therapy treatment. Radiat Environ Biophys 53(2): 381-390.

Stiller C (2007) Childhood cancer in Britain: incidence, survival and mortality. Oxford Unviersity Press: Oxford, UK.

Tucker M, Meadows AT, Boice JD, Stovall M, Oberlin O, Stone BJ, Birch J, Voute PA, Hoover RN, Fraumeni JF (1987) Leukemia after therapy with alkylating agents for childhood cancer. J Natl Cancer Inst 78(3): 459-469.

Zeltzer LK, Lu Q, Leisenring W, Tsao JC, Recklitis C, Armstrong G, Mertens AC, Robison LL, Ness KK (2008) Psychosocial outcomes and health-related quality of life in adult childhood cancer survivors: a report from the childhood cancer survivor study. Cancer Epidemiol Biomarkers Prev 17(2): 435-446.

(c) (i) This work is licensed under the Creative Commons Attribution 4.0 International License. To view a copy of this license, visit http://creativecommons.org/licenses/by/4.0/

Supplementary Information accompanies this paper on British Journal of Cancer website (http://www.nature.com/bjc) 